\title{
MeV Ultrafast Electron Scattering at SLAC: Status and Opportunities
}

Renkai Li, Suji Park, Xiaozhe Shen, Stephen Weathersby, Jie Yang and Xijie Wang SLAC National Accelerator Laboratory, 2575 Sand Hill Road, Menlo Park, CA 94025, USA

Electron scattering, including diffraction, imaging and spectroscopy, techniques are indispensable research tools for new discoveries in material science, chemistry, biology, as well as for new technology breakthroughs for industrial applications. A new development in the electron scattering family is the use of femtosecond $\mathrm{MeV}$ electrons for studying photoinduced non-equilibrium processes. In the last decade, there has been significant worldwide R\&D efforts on femtosecond time-resolved $\mathrm{MeV}$ electron scattering aiming at reaching ever better temporal, spatial and energy resolutions. SLAC National Accelerator Laboratory is enthusiastically pursuing this research direction.

A MeV ultrafast electron diffraction (UED) system has been built at SLAC, which is now supporting a very active science program, and at the same time serving as a testbed for instrumentation development. In this talk, we will briefly review the design and performance of the machine. A wide range of samples and dynamics, including thin nano-films, hetero-structures, 2D materials, nanoparticles, warm-dense matter, and gas-phase molecules, have been studied. Meanwhile, we are developing new machine capabilities to enable new science opportunities. For example, by generating ultra-low emittance beams and applying strong magnetic focusing, we demonstrated micro-diffraction for measuring $\mu \mathrm{m}$-size samples or domains while maintaining ultrahigh temporal resolution. We recently added $\mathrm{THz}$ pumping capability and we will present results on $\mathrm{THz}$ timestamping of electron beams to enable femtosecond temporal resolution and beyond.

Future developments for UED include better temporal resolution to 10-fs-level and enhanced sample handling capabilities such as heating/cooling, precise rotation control, etc. We are developing key technologies for future UEM with unprecedented combined spatial-temporal resolution based on a superconducting radio-frequency source. We will also discuss a new concept for realizing electron energy-loss spectroscopy using RF gun generated MeV electron beams to study electronic dynamics with sub-eV and 10-fs resolutions. 